\title{
Incorporating Learning and Aging Attributes of Workers into A DES Model
}

\author{
Maji Ibrahim Abubakar \\ School of Engineering \\ University of Portsmouth \\ Portsmouth PO1 3DJ, UK \\ e-mail: up672524@myport.ac.uk
}

\author{
Qian Wang \\ School of Engineering \\ University of Portsmouth \\ Portsmouth PO1 3DJ, UK \\ e-mail: qian.wang@port.ac.uk
}

\begin{abstract}
In manufacturing plants, part of assembly is often performed by human workers and therefore its performance largely depends on humans (or human performances) rather than machines. However, most studies on human centred assembly systems using the modelling simulation methods do not consider or incorporate the effects of human performance that may also impact the overall system performance of such as an assembly line. The prediction of human performance or behaviour in a manufacturing system evaluation is often overlooked by researchers or system designers partially due to a lack of proper versions of existing simulation tools that can incorporate human attributes into an established simulation model. This paper presents a study by incorporating human attributes of learning and ageing into a discrete event simulation (DES) model based on a human centred assembly system. The effects of worker performance due to aging and learning were investigated and identified through a literature review [1]. The simulation result demonstrates that the worker performance may approach his/her full capacity at the age of 38 years old and this may decrease by an average of $1 \%$ from 38 to 40 years old, and $6 \%$ at the age of 45 years old. After the age of 45 years old, the decline rate of worker performance may slow down as it can be offset by accumulating worker experience through learning.
\end{abstract}

Keywords-manufacturing systems; human factors; learning curve; discrete event simulation

\section{INTRODUCTION}

Most products virtually require assembly, which is often performed by human workers. Despite the benefits of automation implemented in manufacturing sectors, manual assembly methods still have some advantages; one of which is that human workers interact easily and respond rapidly to the unpredictable circumstances [2]. For human centred manufacturing systems, a study shows that assembly operations account for more than $53 \%$ of production time or $20 \%$ of unit cost of manufactured products [3]. Nevertheless, human performance is often inadequately addressed, overestimated or simply ignored in terms of manufacturing systems design and evaluation [4].

For instance, a walking worker assembly line is considered as a lean manufacturing system where each worker performs and completes assembly tasks of a product by travelling down the line. Such a system performance largely relies on the performance of individual workers. The main concern of the walking worker line is that the working speed of each worker, who walks and stops at each station to assemble a product, can vary significantly along the line [5]. The individual performance may be affected by human factors including cognitive elements (such as experience) and physical elements (such as age).

There are some studies in a view of socio-technical or psychological sciences to evaluate the effect of human performance relating to the design of manufacturing systems. Nevertheless, these studies are basically described in a form of language that manufacturing engineers often find difficult to understand. In other aspect, most modelling simulation methods in the production research literature are focused on conventional systems in which operators are tied to specific tasks or stations. Moreover, current modelling simulation tools in the market for manufacturing systems evaluation do not provide facilities that allow system designers to combine human attributes (or human performance) into an investigation of the overall system performance. This is because, for example, in a discrete event simulation (DES) model, the workers are defined and treated as a simple resource the same as parts, machines, conveyors and so on [6]. The application of DES simulation models is therefore restricted to predicting such variables as the required number of workers, their shift patterns and routes.

The paper describes a method that was used to evaluate the performance of individual workers by incorporating parameters of varying age and experience (through a learning process) into an integrated DES model. With this approach, the effects of human performance due to aging and experience can be quantified.

\section{EXPERIENCE}

Experience can be defined as the knowledge or a skill to be gained through involvement of a specific task, event or subject. In a process of human centred assembly, individual human performance can be improved by working experience through learning after repeatedly performing an assigned task until the time spent on completion of this task is stabilised. Most of the learning theory developed for manufacturing analysis is based on the mechanisms of skill acquisition and the law of practice [4]. Performance improvement is often measured in terms of a reduction of job cycle time, where the reduction in cycle time is the result of the learning process. Thus, the learning curve theory can be used to measure the job experience of a worker with the parameter $\mathrm{V}(0 \leq \mathrm{V} \leq 1)$, where if $\mathrm{V}=0$ it indicates a task that is manually performed and $\mathrm{V}=1$ it indicates a task that 
is predominantly executed by machines [7]. Hence, time to produce $n^{\text {th }}$ unit is given by:

$$
T_{n}=D \times\left[V \times(1-V) n^{c}\right]
$$

The standard time to complete a task regardless of the cumulative number of units produced is expressed as follows:

$$
\omega=D \times V
$$

where:

$$
\begin{aligned}
& T_{n}: \text { Time to produce } n^{\text {th }} \text { unit } \\
& \omega: \text { Standard time } \\
& D: \text { Time to produce the first unit } \\
& V: \text { Incompressibility factor } \\
& n: n^{\text {th }} \text { unit }
\end{aligned}
$$

$C$ : Learning index which determines the speed of learning occurring each time as the cumulative output increases, it is computed as $\log (R) / \log (2)$ where learning rate $\mathrm{R}$ is measured in percentage $(0<\mathrm{R}<1)$, e.g., $80 \%$ of learning rate $\mathrm{R}$ implies a reduction of $20 \%$ in cost of direct man labour hour needed to complete a subsequent unit.

Figure 1 shows a linear correlation in variation of assembly time against the increase of worker ages, the result was calculated with the correlation coefficient $R=0.99$ in this case.

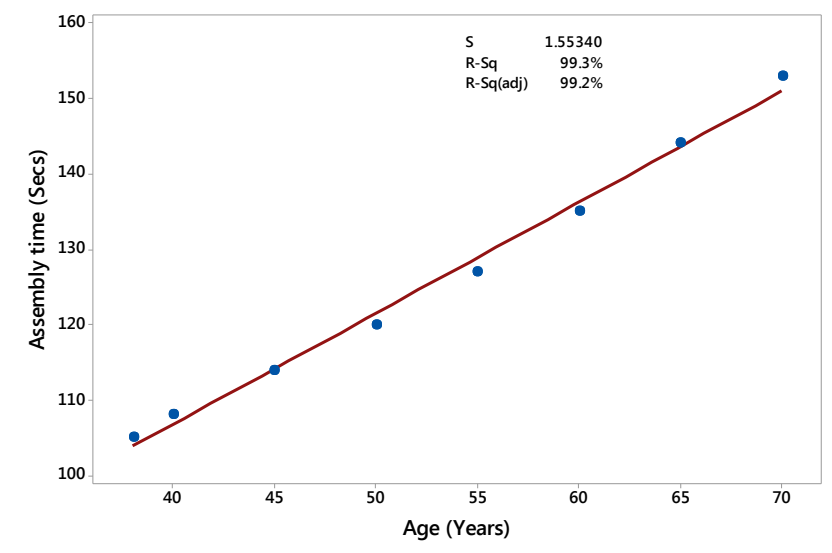

Figure 1. Assembly time of workers under different ages.

\section{AGEING}

As one of human factors, ageing can cause the persistent decline in biological components due to the internal physiological deterioration [8]. This leads to the decline of such as visual ability, musculoskeletal force, flexibility, motion capability, memory or concentration and thermoregulation $[9,10,11]$. In this current study, it was assumed that the natural decline of physical, physiological and cognitive systems over the increase of age is unavoidable. Nevertheless, as worker's age increases, it is expected that the accumulation of his/her experience through learning also increase and this may offset the decline of the human abilities $[11,12,13]$.

Figure 2 shows the trend in the decline of human functional capacity over the increase of age after 38 years old at which the human functional capacity reaches the maximum rate. After this age, the loss rate in percentage can be expressed below [1]:

$$
L_{r}=0.57+0.012 \mathrm{k}
$$

where

$L_{r}=$ Loss rate in percentage

$k=$ Age in years

Thus the remaining capacity in percentage after 38 years old can be given by:

where:

$$
F_{r m}=k_{2}-L_{r}\left(k_{1}-38\right)
$$

$F_{r m}$ : Remaining capacity in percentage after 38 years old $k_{2}$ : Full capacity $(100 \%)$ at 38 years old

$L_{r}:$ Loss rate in percentage

$k_{1}$ : Existing age in years

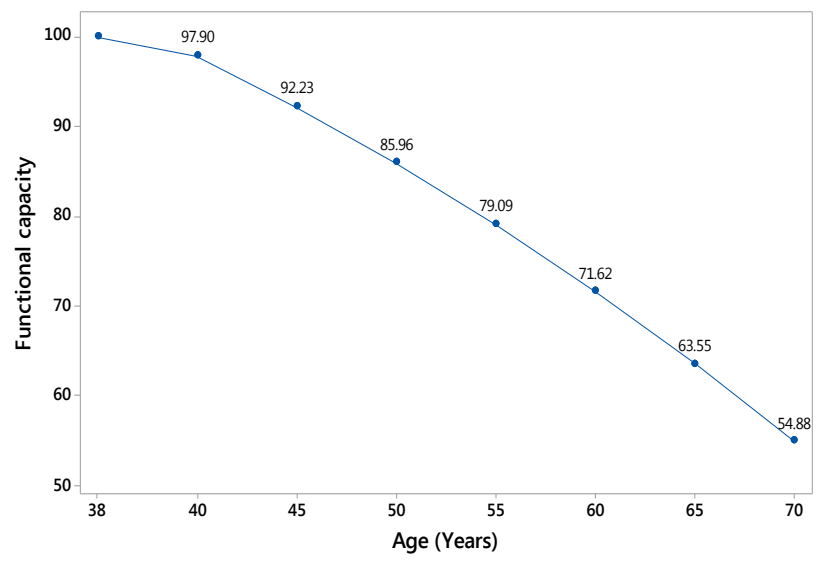

Figure 2. Decline of human functional capacity over the increase of ages of workers.

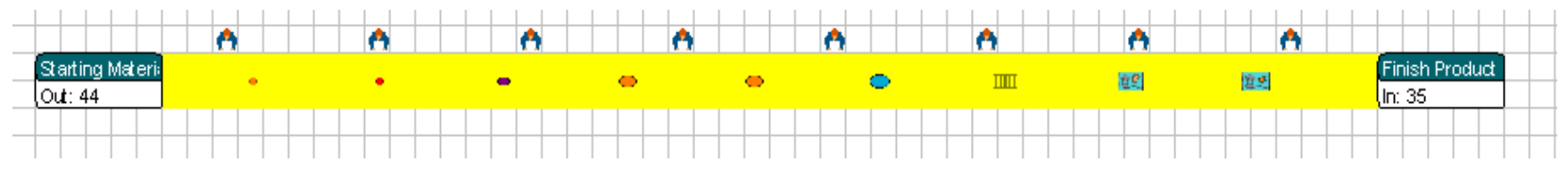

Figure 3 A linear assembly line model operated by workers under different ages. 


\section{SimUlation RESUlTS AND ANALYSIS}

In this case study, a DES model was established using a DES tool called Enterprise Dynamics, which interacts with an external MS Excel worksheet that defines variables of worker age and experience (as described in Section 2 and 3) in order to generate the variation of human performance by individual worker at the different age. Figure 3 shows a human centred assembly line model using the DES tool; the line consists of eight stations manned by eight workers under different ages; 240 units of products were assembled on the line and the average assembly time for each individual worker was examined based on the simulation result.

Figure 4 shows the trend of the average assembly time over the increasing number of assembled units of each worker under different ages. This is also known as experience curve or learning curve, which represents the improved efficiency or performance through learning or practices by repeating operations of a specific task a worker performs. The results were obtained by initially setting a maximum assembly time a worker at 70 years old possibly needs at the beginning of assembling a new product. The average assembly time then declines during a learning process for each worker at the different ages by repetitively performing the same amount of assembly tasks making a unit of the same product. The assembly time finally approaches a steady state after producing 240 units for all workers under the different ages.

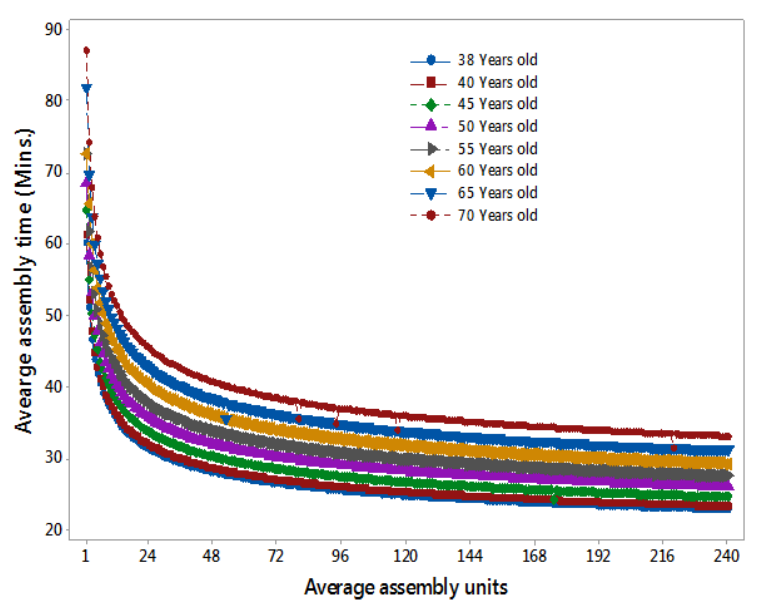

Figure 4. Assembly time vs output in a learning process of workers under different ages.

It can also be seen from Figure 5 that the average assembly time of each worker to produce 240 units increases by $1.38 \%$ at the age from 39 to 40 years old. At the age of 45 years old it rises to $6.44 \%$. After this age, it drops gradually to $4.8 \%$ at the age of 70 years old. This result indicates that the increase of assembly time due to the increase of age of the worker after 45 years old starts to decline as it is offset by the increase of accumulative experience of the worker over the increase of age.

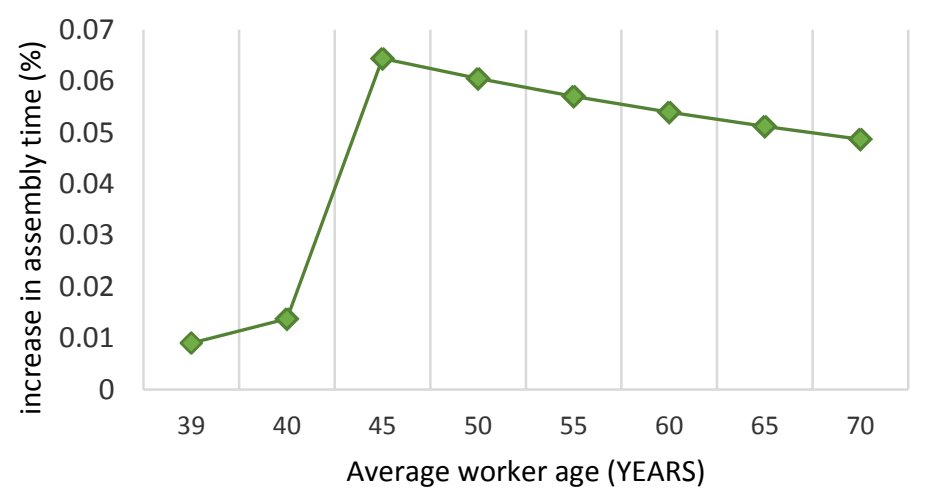

Figure 5. Trend in increase of percentage of assembly time for workers under different ages.

\section{CONCLUSIONS AND DISCUSSIONS}

This paper presents an investigation based on findings of a literature study, which shows that individual performance of human workers usually approaches their full capacity at the age of 38 years old. After this age, it may start to decline over the increase of ages of workers. By contrast, the literature study also indicates the increase of experience over the increase of age of workers can also improve the individual performance that may also compensate the loss of assembly time due to the increase of ages of workers [1]. Furthermore, the effects of experience and ageing on worker performance were examined by incorporating their parameters into a DES tool, which was used for modelling a linear human centred assembly line operated by workers at under varying ages as specified in this case. With this method, the difference of performance of each worker at the different ages can be quantified. The study concluded that the worker performance may reach its full capacity at the age of 38 years old and it decreases by an average of $1 \%$ from 38 to 40 years old, and $6 \%$ at the age of 45 years old. After this age, the decline rate of performance may slow down as it can be compensated by the increase of experience of workers.

On the other hand, existing commercial simulation packages offer only dedicated application object libraries for developing fast and efficient models of common scenarios 
and these tools can be characterised as limited functionalities in the field of manufacturing. The future challenges and trends of simulation tools are to introduce cloud-based technologies in order to facilitate the mobility of the applications and the interoperability between different partners. Currently, only few commercial tools have integrated this function. In addition, efforts are being made towards the creation of application that run in multiple and mobile devices. The extended use of open and cloud-based tools can address these problems and result in high performance computing at a minimum cost.

Another issue is that there is also a lack of proper data exchange among different domains and few or no common standards or integrated frameworks, which cause difficulties in the interoperability and collaboration between systems and partners. Application of building incremental models allows in-process debugging but often this does not work properly and this also increases the complexity of finding out the problems of the developed models. All these issues can be addressed with the development and utilisation of multidisciplinary and multi-domain integrated simulation tools. As far as the lifecycle simulation is concerned, the poverty of adequate modelling tools ought to be noted. Only few applications take into serious considerations of product lifecycle costs and environmental issues. In addition, some modelling tools are usually aimed at the re-manufacturing of specific product types and these tools are still insufficient for de-manufacturing of products. Hence, researchers should focus on the development of tools for the field of lifecycle management. Currently, object-oriented, hierarchical models of plants encompassing business, logistic and production processes exist but the direct integration of modelling tools with CAD, DBMS (ORACLE, SQL Server, Access, etc.) and direct spreadsheet link in/out, XML, HTML reports are still limited. As a result, simulation tools that will assure the multi-level integration among them should be developed as priority. Gradually, enterprises are starting to adopt the concepts and the models of the virtual factory. But, the technologies related to virtual factory especially with data acquisition, control and monitoring are still expensive, complicated and hard to apply. Thus, the research should move towards the direction of real-time factory applicable and affordable tools to be developed. Efforts may also be made to create smart, intelligent and self-learning tools incorporating integrated neural networks and inbuilt algorithms for automated optimisation of system parameters. Moreover, there are applications that base on empirical or past data and some knowledge-based advisory systems.
Although satisfactory and analytical simulation capabilities in continuous processing units can be noticed, research is required in order to develop more intelligent tools that will lead to autonomous and-self adapting systems [6].

\section{ACKNOWLEDGMENT}

The authors would like to thank the industrial participants for their contributions of a survey to this project.

\section{REFERENCES}

[1] M. I. Abubakarand and Q. Wang, "Key human factors and their effects on human centered assembly performance - a literature review based study", International Journal of Industrial Ergonomics, under the second review, 2018 .

[2] V. L. Shalin, G. V. Prabhu and M. G. Helander, "A cognitive perspective on manual assembly", Ergonomics, Vol. 36 (1), pp. 108$127,1996$.

[3] S. N. Samy amd H. Elmaraghy, "A model for measuring product assembly complexity", Internationnal Journal of Integrated Manufacturing, Vol. 23 (11), pp. 1015-1027, 2010.

[4] Q. Wang, M. Sowden and A. R. Mileham, "Modelling human performance within an automotive engine assembly line", International Journal of Advanced Manufacturing Technology, Vol. 68 (1-4), pp. 141-148, 2013.

[5] Q. Wang, S. Lassalle, A. R. Mileham and G. W. Owen, "Analysis of a linear walking worker line using a combination of computer simulation and mathematical modeling approaches", Journal of Manufacturing Systems, Vol.28, No.2-3, pp.64-70, pp. 2009.

[6] Q. Wang and C. R. Chatwin, "Key issues and developments in modelling and simulation-based methodologies for manufacturing system analysis, design and performance evaluation", International Journal of Advanced Manufacturing Technology, Vol. 25, pp. 1254$1265,2005$.

[7] J. R. Dejong, "The effects of increasing skill and its consequences for time standards", Ergonomics, Vol.1, pp. 51-60, 1957.

[8] G. P. Kenny, H. Groeller, R. McGinn and A. D. Flouris, "Age, human performance, and physical employment standards", Applied Physiology Nutrition and Metabolism, Vol. 41 (6), pp. 92-107, 2015.

[9] M. Peruzzini and M. Pellicciari, "A framework to design a humancentred adaptive manufacturing system", Advanced Engineering Informatics, pp. 1-20, 2017.

[10] A. Robertson and C.S. Tracy, "Health and productivity of older workers", Scand. J. Work Environ Health, Vol. 24(2), 85-97, 1998.

[11] P. Warr, "Work and Aging: a European perspective, Great Britain", Tailor and Francis Ltd., 1994.

[12] T. A. Salthaouse and B. L. Somberg, "Skilled performace: effects of adult age and experince on elementary processes", Journal of Experimental Psychology, Vol. 111 (2), pp. 176-207, 1982.

[13] S. Giniger, A. Dispenzieri and J. Eisenberg, "Age, experience, and performance on speed and skill jobs in an applied settings", Journal of Applied Psychology, Vol. 68 (3), pp. 469-475, 1983. 